\title{
Neglect Application for Block the Unwanted Notification
}

Lomesh Pelne lomeshpelne786@gmail.com

Suraj Kale ${ }^{1}$ surajrk.kale85@gmail.com

Rajat Kubde ${ }^{1}$ rajatkubde49@gmail.com

Vrushabh Bhusari ${ }^{1}$ vrushabh38@gmail.com

Vandana Choubey ${ }^{2}$ vandana.dubey611@gmail.com

${ }^{1}$ Student, Department of Computer Engineering, Smt. Radhikatai Pandav College of Engineering, Nagpur, India

${ }^{2}$ Assistant Professor, Department of Computer Engineering, Smt. Radhikatai Pandav College of Engineering, Nagpur, India

\section{INTRODUCTION}

Useless notifications will be blocked smartly and gathered together in one place, so the phone notification bar will always be in a clean and tidy status. The app stops trash notifications from jamming and slowing down your phone, keeping your phone free from disturbing and annoying notifications [1].

Grant notification access permission: notifications access must be enabled for neglect in order to block notifications. When it is disabled, the app will guide you to the settings to enable it. Don't worry, the app will not collect and upload any of your privacy in the notifications [3].

Research Article

First Online on - 19 June 2021

(C) 2021 RAME Publishers

This is an open access article under the CC BY 4.0 International License

https://creativecommons.org/licenses/by/4.0/

Cite this article - Lomesh Pelne, Suraj Kale, Rajat Kubde, Vrushabh Bhusari, Vandana Choubey, "Neglect Application for Block the Unwanted Notification", International Journal of Computational and Electronic Aspects in Engineering, RAME Publishers, vol. 2, issue 2, pp. 24-27, 2021

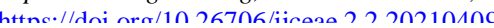

Find blocked notifications: all of the junk notifications are put together in the neglect app; click neglect on the launcher and then in navigation bar click on notification and find all unread junk notifications [4].

Hide app from launcher: now a days there are many phones who gives a feature of hide an application from a launcher but if you want to open the application then you have to first unhide and then you can open it. Neglect has its own features to hide app icon from launcher and no need to unhide the neglect to open you can open it from the phone dialer. You need to just dial a specific number in dialer and then neglect app open [1].

\section{METHODS}

In past, people don't have application and smartphones so that they can block notification of other application. They were using different methods like offline silent phone, disconnect internet [2]. That time if some important mail or message comes then it will be miss by the user by using these app users can block notification as well as it will read all 
blocked notification later [3]. But, at present the technology has reached the zenith with beautiful software and application with perfect prediction which is helping the people in day-to-day life so that people can do their work easily and during work time they can block un-necessary notification [3].

Now, there are ample amount of application for this feature like dnd, app block, google and many hundreds of applications you can see in play store. So, as a beginner $i$ have developed this neglect application which will help the people for blocking unnecessary notification while doing some work [4].

\section{A. Aim}

Push notifications containing ads are pretty common, but thankfully, they're usually easy to get rid of. On any phone running android 5.0 lollipop or higher, just press down on the notification to see which app it came from. Most of the time, the app name is already visible at the top of the alert, but some apps hide that detail [3]. You'll probably want to uninstall the offending app, but if you want to keep it, you can choose to just hide the notifications [4]. After you hold down on the notification, there should be an option to block future alerts from that app [1].

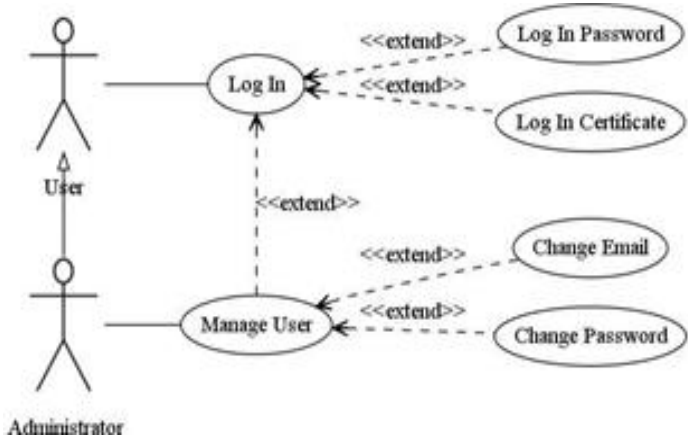

Figure 1: Case diagram

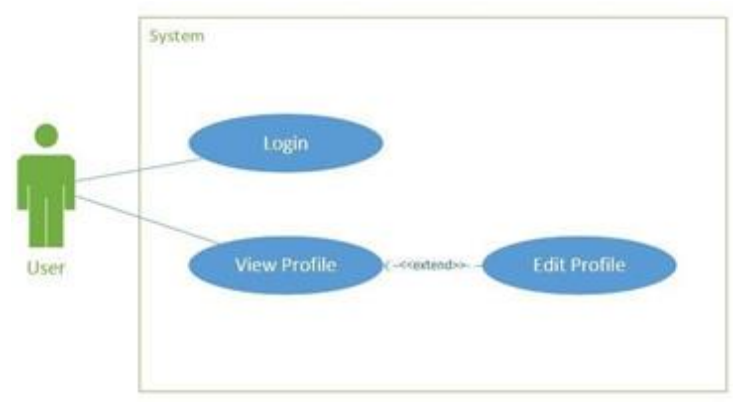

Figure 2: Neglect App Module Diagram

\section{B. System requirements}

- Operating system: windows 7/8/10.

- Platform : android studio 3.3,

- Back end : java, html and xml,sqlite database ,services, broadcaster receiver

- Processor : intel core i3, intel core i5,intel core i7.

- Hard disk : $40 \mathrm{gb}$.

- Ram : $4 \mathrm{gb}$

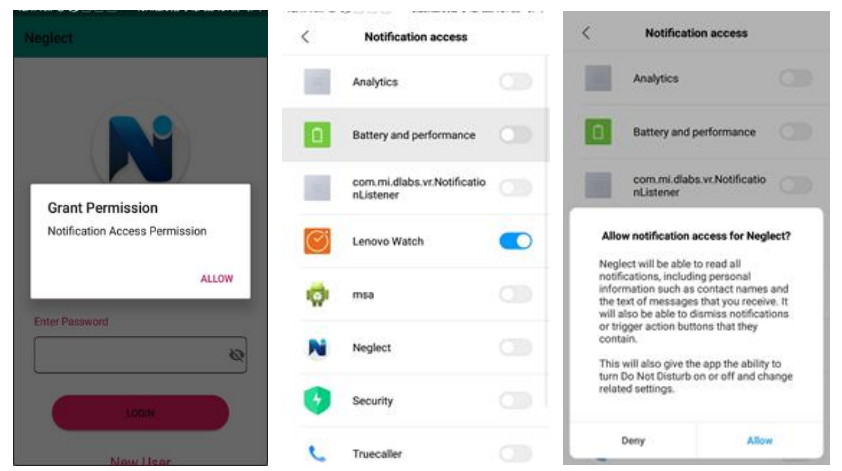

Fig 1: Permission activity

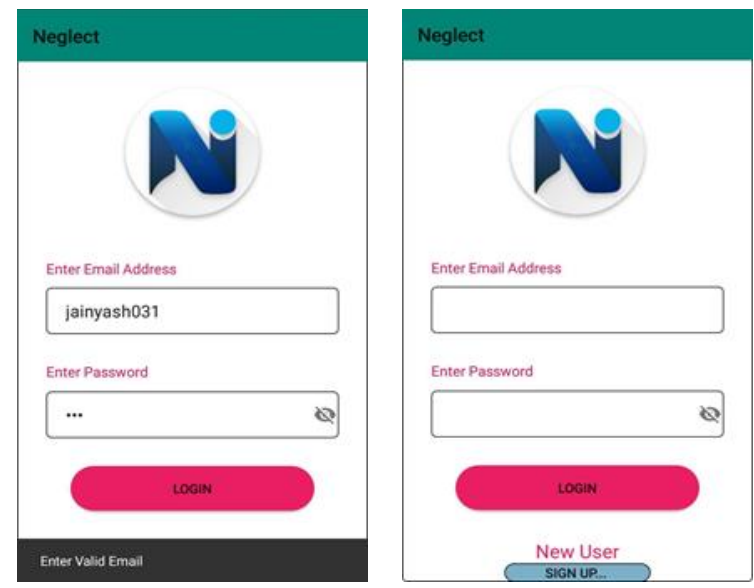

a. Login activity

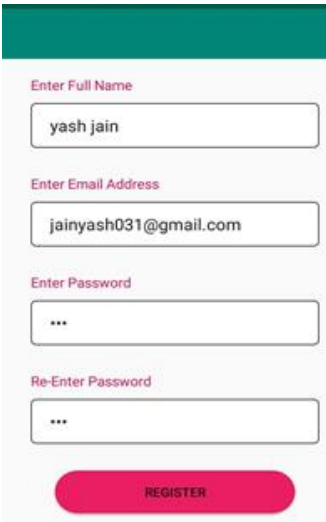

b. Sign up activity

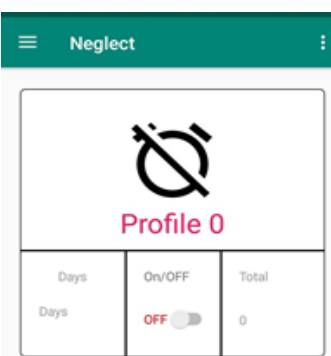

Figure 2: login activity \& sign-up activity \&. Main activity 


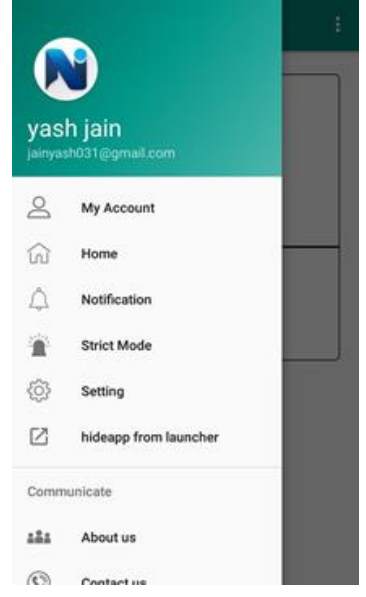

navigation drawer

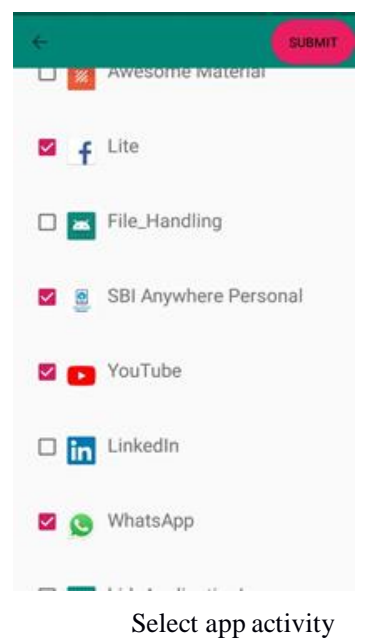

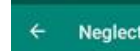

Name

silent

Days

\section{2}

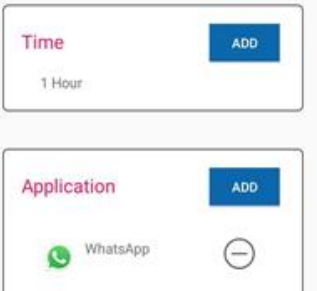

Set profile

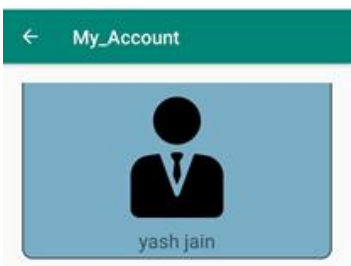

Name

\section{yash jain}

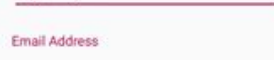

jainyash031@gmail.com

Change Password

...

M y account activity

Fig 3: navigation \& set profile \&.select app activity \& my account activity

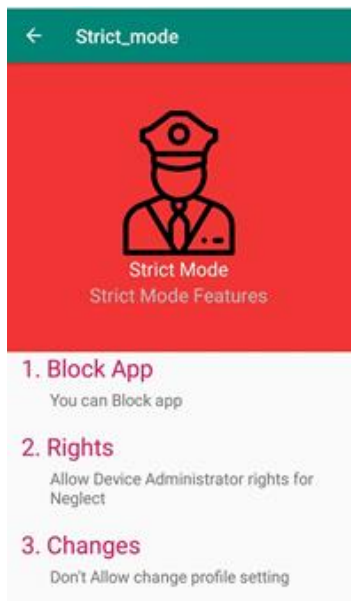

$\leftarrow$ NotificationNav_bar $\quad$ :

All Blocked Notification Shown Here:

(1) Notification Send

My Pending Notification

Notification blocked activity: also shows all the blocked notification in application it self. As a beginner ihave developed this application with the great support from my team leader miss [4]. Vandana mam. This application is still under construction because for more additional features which can be useful for the people in an easier approach [2].

\section{B. Feasibility analysis}

Neglect is simple $5 \mathrm{mb}$ compatibility version android 4.1 (jelly bean) it needs server and internet facilities for running the application. It is properly designed based on study features for the user. You can download this application and enjoy the features . And the application is still on developing so it will take some time to fix the bugs, which will make the users satisfaction $[4,5]$.

\section{PROJECT LEGACY}

\section{A. Technically}

First of all, additional skills in the java programming language are needed which is somewhat difficult for user. Also use a lot of components in android studio is learned such as the debugging [1]. A new language basics $\mathrm{xml}$ is also learned, which is not known at all earlier

\section{B. Managerial lesson}

There are lot of human things in this placement and project. First of all, with regard to the user [2]. There are lot of autonomy to acquire. Indeed, we did not already know anything about android, but had a small interest in knowing "how this app works" and learned a new skill with that interest [3]. The trial process has been carried out in the current session and going to work with android because of beginner and want to change from a developer to a new developer. [4,5].

\section{CONCLUSION}

Push notifications can be focused on the particular segments of your client base, and even can be customized for particular application clients. This is a noteworthy advantage when contrasted with SMS text messaging be that as it may, these website push notifications additionally

\section{A. Product definition}

Neglect is simple $5 \mathrm{mb}$ sized application designed by me which will help the people to block the notification [3]. It 
require the management of user identification data along with some sort of interface for composing messages, focusing on them and sending them to ensure guaranteed conversions. Publishers can manufacture this framework themselves, or they can enlist a vendor to build it for them. Progressively, application distributers/publishers pay for these administrations.

\section{Future Scope}

Futures provide a way to reason about performing many operations in parallel- in an efficient and non-blocking way. A future is a placeholder object for a value that may not yet exist. Generally, the value of the future is supplied concurrently and can subsequently be used. Composing concurrent tasks in this way tends to result in faster, asynchronous, non-blocking parallel code.

\section{References}

[1] Shamsi T. Iqbal and Eric Horvitz., "Notifications and awareness: a field study of alert usage and preferences",
Proceedings of the 2010 ACM Conference on Computer Supported Cooperative Work, CSCW 2010, Savannah, Georgia, USA, February 6-10, 2010.

[2] Leiva, L., B" ohmer, M., Gehring, S., and Kruger, A., "Back to the app: the costs of mobile application interruptions", MobileHCI'12, September 21-24, 2012, San Francisco, CA, USA.

[3] Oulasvirta, A., Rattenbury, T., Ma, L., and Raita, E., "Habits make smartphone use more pervasive", Personal and Ubiquitous Computing volume 16, pages105-114, 2012.

[4] McMillan, D., Morrison, A., Brown, O., Hall, M., and Chalmers, M., "Further into the wild: Running worldwide trials of mobile systems", Lecture Notes in Computer Science book series, LNCS, volume 6030, Pervasive Computing pp 210-227, 2010.

[5] Wiese, J., Saponas, T. S., and Brush, A., "Phoneprioception: enabling mobile phones to infer where they are kept", Proceedings of the SIGCHI Conference on Human Factors in Computing Systems, April 2013 Pages 2157-2166.. https://doi.org/10.1145/2470654.2481296. 